\title{
Concerted Upregulation of CLP36 and Smooth Muscle Actin Protein Expression in Human Endometrium during Decidualization
}

\author{
Ulrich Miehe $^{a}$ Peruka Neumaier-Wagner $^{a} \quad$ Mamed Kadyrov $^{b}$ Pankaj Goyal ${ }^{d}$ \\ Joachim Alfer ${ }^{c}$ Werner Rath ${ }^{a}$ Berthold Huppertz ${ }^{b}$ \\ Departments of ${ }^{a}$ Obstetrics and Gynecology, ${ }^{b}$ Anatomy and ${ }^{c}$ Pathology, University Hospital RWTH Aachen, \\ Aachen, and ${ }^{d}$ Institute for Prevention of Cardiovascular Diseases, University of Munich, Munich, Germany
}

\section{Key Words}

Actin - CLP36 - Cytoskeleton - Decidualization ·

Pregnancy

\begin{abstract}
The human endometrium prepares for implantation of the blastocyst by reorganization of its whole cellular network. Endometrial stroma cells change their phenotype starting around the 23rd day of the menstrual cycle. These predecidual stroma cells first appear next to spiral arteries, and after implantation these cells further differentiate into decidual stroma cells. The phenotypical changes in these cells during decidualization are characterized by distinct changes in the actin filaments and filament-related proteins such as $\alpha$-actinin. The carboxyterminal LIM domain protein with a molecular weight of $36 \mathrm{kDa}$ (CLP36) is a cytoskeletal component that has been shown to associate with contractile actin filaments and to bind to $\alpha$-actinin supporting a role for CLP36 in cytoskeletal reorganization and signal transduction by binding to signaling proteins. The expression patterns of CLP36, $\alpha$-actinin and actin were studied in endometrial stroma cells from different stages of the menstrual cycle and in decidual stroma cells from the 6th week of gestation until the end of pregnancy. During the menstrual cycle, CLP36 is only expressed in the luminal and glan-
\end{abstract}

dular epithelium but not in endometrial stroma cells. During decidualization and throughout pregnancy, a parallel upregulation of CLP36 and smooth muscle actin, an early marker of decidualization in the baboon, was observed in endometrial decidual cells. Since both proteins maintain a high expression level throughout pregnancy, a role of both proteins is suggested in the stabilization of the cytoskeleton of these cells that come into close contact with invading trophoblast cells.

Copyright $\Subset 2005$ S. Karger AG, Basel

\begin{tabular}{ll}
\hline Abbreviations used in this paper \\
\hline ALP & actinin-associated LIM protein \\
Clik-1 & CLP36-linked kinase 1 \\
CLP36 & $\begin{array}{l}\text { carboxy-terminal LIM domain protein with a } \\
\text { decidual stroma cell }\end{array}$ \\
DSC & endometrial stroma cell \\
ESC & extravillous trophoblast cell \\
EVT & glandular epithelial cell \\
glEC & Lin11, Isl-1, Mec-3 \\
LIM & luminal epithelial cell \\
luEC & PSD95, DLG, ZO-1 \\
PDZ & precursor cells of the decidual stroma cells \\
preDSC & smooth muscle actin \\
sm-actin &
\end{tabular}

\section{KARGER}

Fax +4161306 1234 E-Mail karger@karger.ch www.karger.com
Dr. Berthold Huppert

Department of Anatomy II, University Hospital RWTH Aachen Wendlingweg 2

DE-52057 Aachen (Germany)

Tel. +49 2418089 975, Fax +49 2418082 472, E-Mail bhuppertz@ukaachen.de 


\section{Introduction}

In preparation for implantation of the blastocyst, decidual tissues consist of a specialized network of cells derived from the endometrium. This cellular network is composed of at least four different cell types: epithelial cells (luminal and glandular), leukocytes, endothelial cells and decidual stroma cells. The latter arise from proliferation and differentiation of endometrial stroma cells under the influence of progesterone [Kimatrai et al., 2003]. Endometrial stroma cells are fibroblast-like cells which increase in size and store glycogen and lipids starting on day 23 of the menstrual cycle. Thus endometrial stroma cells achieve an epithelioid phenotype and at that stage of the cycle are called predecidual stroma cells. The latter cells first appear next to the spiral arteries and later build a dense cellular stroma underlying the luminal epithelium [Noyes et al., 1950]. As soon as implantation is successful and pregnancy starts, these cells are called decidual stroma cells.

The morphological changes during decidualization are accompanied by distinct changes in the composition of the cytoskeleton, including changes in microfilaments (actin) and intermediate filaments such as vimentin and desmin, as well as filament-related proteins such as $\alpha$-actinin. Furthermore, proteins such as cadherins and integrins that are involved in cell-cell and cell-matrix interactions and that are attached to the cytoskeleton undergo dramatic changes in endometrial cells during the menstrual cycle and during differentiation of endometrial stroma cells into decidual stroma cells [MacCalman et al., 1996, Getsios et al., 1998, Yoshimura et al., 1998]. Finally, decidual stroma cells achieve a contractile myofibroblast-like phenotype characterized by the expression of smooth muscle actin and desmin [Glasser and Julian, 1986, Oliver et al., 1999].

The changes at the morphological level are paralleled by changes in function and protein expression. In cycling human endometrium, decidual cells show a sequence of expression and secretion with relaxin first, then prolactin and finally insulin-like growth factor binding protein 1 [Salamonsen et al., 2003]. All these changes are triggered by external factors such as progesterone or cAMP.

The carboxy-terminal LIM domain protein with a molecular weight of $36 \mathrm{kDa}$ (CLP36) is a cytoskeletal component that has been shown to associate with contractile actin filaments. CLP36 belongs to the family of enigma proteins that contain a C-terminal Lin-11, Isl-1, Mec-3 (LIM) domain [Jurata and Gill, 1998] and an N-terminal PSD95, DLG, ZO-1 (PDZ) domain [Harris and Lim, 2001]. PDZ domains are one of the most common pro- tein-interaction domains that are involved in signal transduction and in clustering transmembrane proteins [Fanning and Anderson, 1999]. In addition, many PDZ proteins interact with the cortical cytoskeleton. PDZ proteins bind to actin directly (e.g. afadin) [Mandai et al., 1997] or to other actin-binding proteins, e.g. binding of actininassociated LIM protein (ALP) [Xia et al., 1997].

The proteins belonging to the enigma family comprise enigma, ENH and cypher/ZASP/oracle, each of which contains three LIM domains [Wu and Gill, 1994; Kuroda et al., 1996; Faulkner et al., 1999], and Ril, ALP and CLP36, each with one LIM domain only [Kiess et al., 1995; Wang et al., 1995; Xia et al., 1997]. They all interact with the cytoskeleton, and studies have suggested that these proteins play a crucial role in the cytoskeletal reorganization and signal transduction of a variety of cells by binding to signaling proteins and the cytoskeleton. Enigma binds to $\beta$-tropomyosin while the others have been shown to associate with $\alpha$-actinin [Xia et al., 1997; Guy et al., 1999; Zhou et al., 1999; Cuppen et al., 2000; Nakagawa et al., 2000; Vallenius et al., 2000, 2004].

CLP36 has been demonstrated to bind to $\alpha$-actinin- 1 in resting platelets and upon platelet activation, CLP36 translocates as a CLP36/actinin complex to the newly formed actin cytoskeleton [Bauer et al., 2000]. Moreover, high expression of CLP36 was found in epithelial cells where it is localized to actin stress fibers via association with $\alpha$-actinin-1 and -4 [Vallenius et al., 2000]. CLP36 colocalizes with $\alpha$-actinin- 1 at cell-cell contacts in endothelial cells and with the muscle-specific $\alpha$-actinin-2 at cell-cell adhesions in the human myocardium [Bauer et al., 2000; Kotaka et al., 2000].

To investigate changes in the CLP36-related cytoskeleton during differentiation of endometrial to decidual stroma cells, the expression patterns of CLP36 and $\alpha$-actinin were studied in endometrial stroma cells from different stages of the menstrual cycle and in decidual stroma cells from the 6th week of gestation until the end of pregnancy. These expression patterns were compared with that of smooth muscle actin, an early marker of decidualization in the baboon [Kim et al., 1998]. The intermediate filament protein vimentin was used as a control determinant for the mesenchymal origin of decidual cells.

\section{Materials and Methods}

\section{Materials}

The study was performed on 43 archival tissue samples obtained from women hospitalized at the Department of Obstetrics and Gynecology of the University Hospital RWTH Aachen, Ger- 
many, during the years 2000-2003. All materials were obtained from the Department of Pathology of the University Hospital RWTH Aachen. Informed consent was obtained from each woman, according to and by approval of the Ethical Committee of the Medical School of the University of Aachen.

Hysterectomized uterine material of 10 normally menstruating women (mean age, 41 years; range, 32-49 years) with large subserous or intraligamentary leiomyomas abutting toward the peritoneal cavity was chosen. Immediately after surgical removal, a representative sample of the normal uterine wall was taken and processed for formaldehyde fixation. Tissues were dated for the stage of the menstrual cycle according to histological criteria. Among these samples, four were dated as proliferative endometrium, and six were dated as secretory endometrium.

From a total of 33 pregnant women (mean age, 29 years; range, 15-39 years), at least one sample of every gestational week ranging from 6 to 25 postmenstrual weeks of gestation, and one of the 33rd and 38th-40th week, respectively, were taken, and either a specimen of the decidua basalis was obtained at the time of pregnancy termination by Novak curette or the placenta with the attached basal plate was obtained after delivery and used for paraffin embedding. These women underwent suction termination of pregnancy for psychosocial reasons $(n=10)$, or underwent induction of delivery because of premature rupture of membranes or intrauterine fetal death $(n=6)$, fetal chromosomal malformation (trisomy 13 or $21 ; n=6$ ) or other fetal abnormalities (congenital muscular dystrophy, left ventricular hypoplasia or skeletal deformity; $n=7$ ) and primary cesarean section because of breech presentation $(n=1$, 33rd week). Placentas of the 38th-40th week were taken from uncomplicated pregnancies after delivery $(n=3)$.

\section{Immunohistochemistry}

A sample block of each tissue $(20 \times 20 \times 5 \mathrm{~mm})$ was fixed in neutral phosphate-buffered $4 \%(\mathrm{v} / \mathrm{v})$ formaldehyde solution for a maximum of $24 \mathrm{~h}$. The samples were dehydrated in a graded series of ethanol and embedded in paraffin (melting point $52^{\circ} \mathrm{C}$; Merck, Darmstadt, Germany). Serial sections $(5 \mu \mathrm{m})$ were cut, mounted on glass slides and deparaffinized using xylene and a graded series of ethanol (10 min each step).

Immunohistochemical staining with monoclonal and polyclonal primary antibodies was performed using a standardized sequence based on the streptavidin-biotin technique for the detection of a biotinylated link antibody. The polyclonal anti-CLP36 rabbit immune serum (dilution 1:300) was developed and kindly provided by the Institute for Prevention of Cardiovascular Diseases, University of Munich, Germany [Bauer et al., 2000]. The monoclonal mouse anti-actinin antibody, AT 6/172 (dilution 1:200, using microwave pretreatment), was obtained from Chemicon International (Hofheim, Germany). The monoclonal mouse anti-actin antibody, B4 (dilution 1:500), was from ICN Biomedical (Costa Mesa, Calif., USA), the monoclonal mouse anti-vimentin antibody, V9 (dilution 1:70), was purchased from Dako (Glostrup, Denmark). Negative control reactions omitting the primary antibodies or using negative controls for polyclonal antibodies (rabbit immunoglobulin fraction; Dako X0936) or monoclonal antibodies (IgG1-negative control antibody; Dako DAK-GO1) displayed no staining. Following immunohistochemical staining of the sections, the staining pattern was evaluated, and a qualitative scoring system was applied differentiating between different cell types.

CLP36 Expression Increases during

Decidualization

\section{Results}

\section{CLP36}

In endometrium of the proliferative $(n=4)$ and secretory phase $(n=6)$, CLP36 immunohistochemistry revealed a weak staining of the luminal and glandular epithelium (fig. 1A, B). Endometrial stroma cells were not stained. In decidual tissues from weeks 6 to 40 of pregnancy $(n=33)$, large round-to-ovoid decidual stroma cells close to the uterine lumen or inside the basal plate of the placenta were positively stained for CLP36 (fig. 1C, D). The same tissue areas were positive for vimentin, indicating that these cells are of mesenchymal origin (fig. 1C, D, inserts).

\section{$\alpha$-Actinin}

In endometrial stroma cells, little staining was detectable in the proliferative phase (fig. 2A). In the late secretory phase, endometrial stroma cells were weakly stained for $\alpha$-actinin (fig. 2B). The brush border of the luminal epithelium was strongly stained in the proliferative phase (fig. 2A), but not during the secretory phase and early pregnancy (fig. 2B, C). In pregnancy, decidual stroma cells were very weakly stained (fig. 2C, D).

\section{Smooth Muscle Actin}

In endometrium of the proliferative and secretory phase, smooth muscle actin immunohistochemistry revealed staining of the luminal epithelium (fig. 3A, B). Stroma cells were weakly stained. In decidual tissues from weeks 6 to 40 of pregnancy, decidual stroma cells close to the uterine lumen or inside the basal plate of the placenta were positively stained for smooth muscle actin (fig. $3 \mathrm{C}$; D). The same tissue areas were positive for vimentin, indicating that these cells are of mesenchymal origin (fig. $3 \mathrm{C}, \mathrm{D}$, inserts).

A qualitative analysis of the staining patterns of the three proteins in the various cell types of endometrium and decidua is shown in table 1.

\section{Discussion}

Our immunohistochemical study demonstrates that CLP36 is strongly expressed in decidual stroma cells during pregnancy, but not in endometrial or predecidual stroma cells. This expression pattern is paralleled by the pattern of smooth muscle actin, which shows a similar staining pattern compared to CLP36. The decidual stroma cells are also slightly positive for $\alpha$-actinin. 

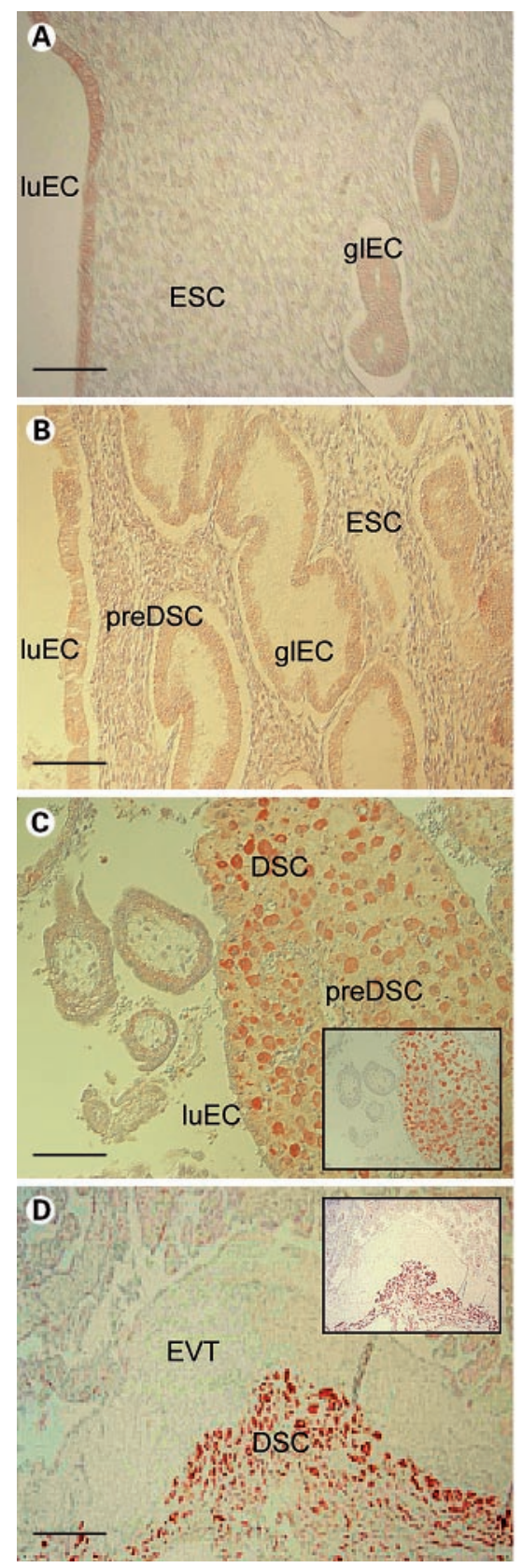

2
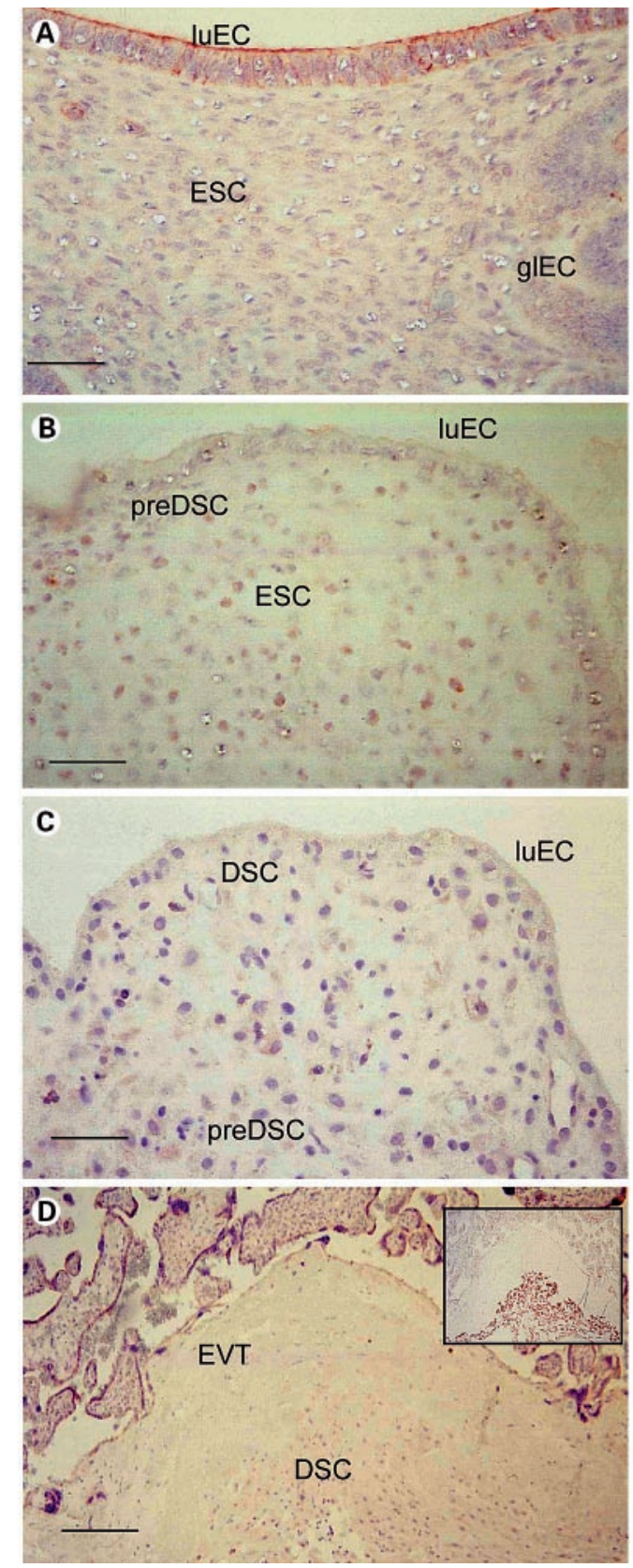

3
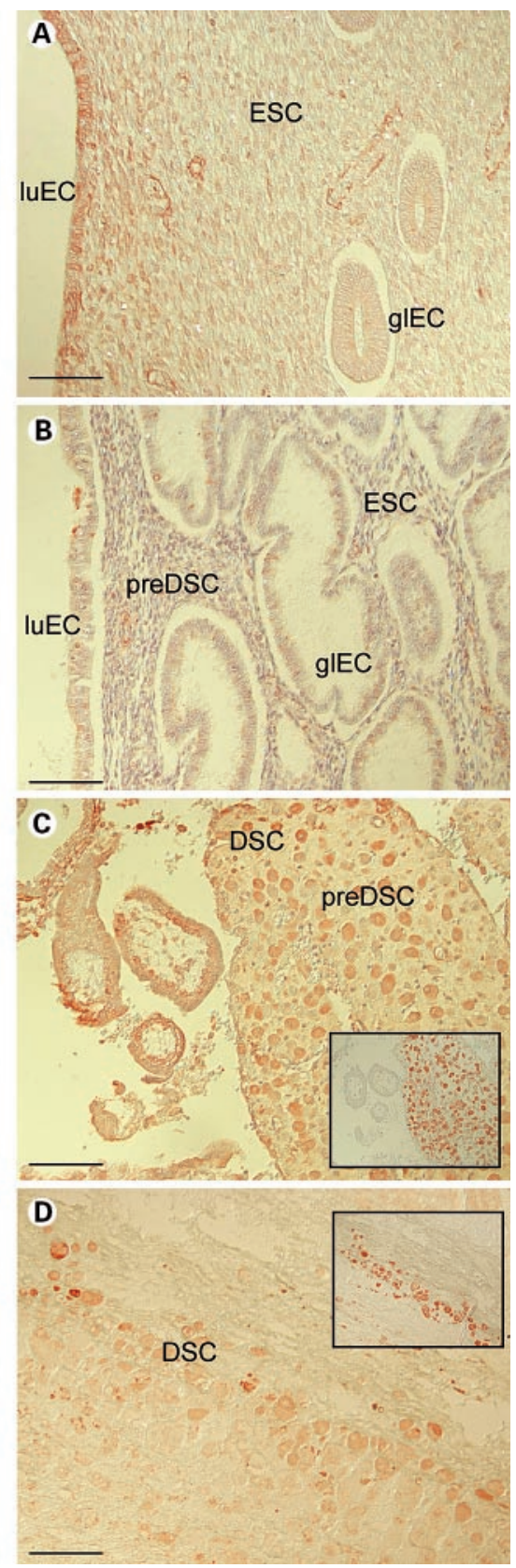

Fig. 1-3. CLP36 (1), actinin (2) and smooth muscle actin (3) immunohistochemical staining of human endometrium of proliferative phase (A), secretory phase (B) and of decidual tissue at 6 (C) and 38 weeks of gestation (D). The inserts show immunohistochemical staining in adjacent sections using a vimentin antibody. A pos-

During the menstrual cycle, CLP36 is only expressed in the epithelia of the uterus, i.e. luminal and glandular epithelium. Expression of CLP36 in endothelial cells [Bauer et al., 2000] and human colon epithelium as well as mouse epidermis [Vallenius et al., 2000] has been de- itive result is shown by red staining. Scale bars $=10 \mu \mathrm{m}$. ESC $=$ Endometrial stroma cells; preDSC $=$ precursor cells of the decidual stroma cells; DSC = decidual stroma cells; EVT = extravillous trophoblast; luEC = luminal epithelial cells; glEC = glandular epithelial cells. scribed before while localization of CLP36 in reproductive organs has not been reported previously. In endothelial cells, Bauer et al. [2000] have shown that CLP36 is associated with stress fibers and is involved in migration and contraction of endothelial cells by directing $\alpha$-ac- 
Table 1. Immunohistochemical localization of CLP36, $\alpha$-actinin, and smooth muscle actin in the non-pregnant human endometrium and in decidua

\begin{tabular}{|c|c|c|c|c|c|}
\hline \multirow{2}{*}{$\begin{array}{l}\text { Endometrial } \\
\text { cells }\end{array}$} & \multirow[t]{2}{*}{ Antibody } & \multicolumn{2}{|c|}{ Stage during menstrual cycle } & \multicolumn{2}{|l|}{ Gestational stage } \\
\hline & & $\begin{array}{l}\text { proliferative } \\
\mathrm{n}=4\end{array}$ & $\begin{array}{l}\text { secretory } \\
n=6\end{array}$ & $\begin{array}{l}6 \text { th-12th week } \\
\mathrm{n}=8\end{array}$ & $\begin{array}{l}13 \text { th-39th week } \\
n=24\end{array}$ \\
\hline \multirow[t]{3}{*}{ ESC } & CLP36 & - & - & 0 & 0 \\
\hline & $\alpha$-actinin & - & $-1+$ & 0 & 0 \\
\hline & sm-actin & $-/+$ & - & 0 & 0 \\
\hline \multirow[t]{3}{*}{ preDSC } & CLP36 & 0 & + & + & 0 \\
\hline & $\alpha$-actinin & 0 & + & - & 0 \\
\hline & sm-actin & 0 & + & + & 0 \\
\hline \multirow[t]{3}{*}{$\mathrm{DSC}$} & CLP36 & 0 & 0 & ++ & ++ \\
\hline & $\alpha$-actinin & 0 & 0 & $-1+$ & $-/+$ \\
\hline & sm-actin & 0 & 0 & ++ & + \\
\hline \multirow[t]{3}{*}{ luEC } & CLP36 & $-/+$ & $-/+$ & - & 0 \\
\hline & $\alpha$-actinin & + & - & - & 0 \\
\hline & sm-actin & + & $-/+$ & - & 0 \\
\hline \multirow[t]{3}{*}{ glEC } & CLP36 & $-/+$ & $-1+$ & - & 0 \\
\hline & $\alpha$-actinin & - & - & - & 0 \\
\hline & sm-actin & - & - & - & 0 \\
\hline
\end{tabular}

If a cell type is not present at a specific stage (non-pregnant or during gestation), it is marked with a zero. $\mathrm{ESC}=$ Endometrial stroma cells; preDSC $=$ precursor cells of the decidual stroma cells; DSC = decidual stroma cells; luEC = luminal epithelial cells; glEC = glandular epithelial cells; sm-actin $=$ smooth muscle actin; $-=$ negative; $-/+=$ weakly positive; $+=$ moderately positive; $++=$ strongly positive.

tinin-1 to specific actin structures such as stress fibers. Stress fibers have been proposed to be involved in the assembly of tight junctions in epithelial cells by tethering of tight junction strands via active actin polymerization to form functional tight junctions [Rajasekaran and Rajasekaran, 2003]. Thus CLP36 might have functional associations with these processes in epithelial cells.

Human endometrial fibroblasts undergo remarkable changes in size and shape as well as in organelle distribution during their transformation into large, round-toovoid decidual cells [Can et al., 1991]. This process begins just prior to blastocyst implantation and is maintained throughout pregnancy. An increase in protein synthesis is paralleled by an increased number of free ribosomes, Golgi cisternae and dilated cisternae of the endoplasmic reticulum [Can et al., 1991].

Vimentin is one example of an intermediate filament protein that is expressed in human endometrial and decidual stroma cells [Franquemont et al., 1991; Can et al., 1995]. Expression of vimentin increases during decidualization, and it persists during pregnancy [Glasser and Julian, 1986].

CLP36 Expression Increases during Decidualization
Recently, it has been demonstrated that human decidual stroma cells express $\alpha$-smooth muscle actin and show similarities with myofibroblasts [Oliver et al., 1999]. The contractile activity of decidual stroma cells is inducible by transforming growth factor- $\beta$, platelet-derived growth factor and interleukin-2, thus supporting the theory of myofibroblast-related cells [Kimatrai et al., 2003]. Contractile stress fibers are composed of about 20 proteins including $\alpha$-smooth muscle actin, actinin, vinculin, vimentin, and pan-myosin [Katoh et al., 1998]. CLP36 is localized to actin stress fibers via binding to $\alpha$-actinin [Vallenius et al., 2000] and therefore, might be one of the remaining unidentified proteins of stress fibers.

Since CLP36 is colocalized with $\alpha$-actinin at cell-cell contacts in endothelial and myocardial cells it might have a regulatory function at cell junctions by binding the CLP36-linked kinase 1, Clik1, to the cytoskeleton. However, the target protein of Clik1 on the cytoskeleton has not yet been identified. Since CLP36 protein expression shows a similar pattern as smooth muscle actin and persists throughout pregnancy, its function may be associated with the stabilization of cell integrity in decidual 
stroma cells rather than playing a role in their differentiation. Our results support the hypothesis of a dynamic regulation of cytoskeletal elements during decidualization and an essential function of the development of a specialized cytoskeleton in decidual stroma cells.

\section{Acknowledgments}

We thank Dr. Joyce Cheung for critically reading and helpful comments on the manuscript.

\section{References}

Bauer, K., M. Kratzer, M. Otte, K.L. de Quintana, J. Hagmann, G.J. Arnold, C. Eckerskorn, F. Lottspeich, W. Siess (2000) Human CLP36, a PDZ-domain and LIM-domain protein, binds to $\alpha$-actinin- 1 and associates with actin filaments and stress fibers in activated platelets and endothelial cells. Blood 96: 4236-4245.

Can, A., M. Tekelioglu, K. Biberoglu (1991) Structure of premenstrual endometrium in $\mathrm{HMG}+$ $\mathrm{HCG}$ induced anovulatory women. Eur J Obstet Gynecol Reprod Biol 42: 119-129.

Can, A., M. Tekelioglu, A. Baltaci (1995) Expression of desmin and vimentin intermediate filaments in human decidual cells during first trimester pregnancy. Placenta 16: 261-275.

Cuppen, E., M. van Ham, D.G. Wansink, A. de Leeuw, B. Wieringa, W. Hendriks (2000) The zyxin-related protein TRIP6 interacts with PDZ motifs in the adaptor protein RIL and the protein tyrosine phosphatase PTP-BL. Eur J Cell Biol 79: 283-293.

Fanning, A.S., J.M. Anderson (1999) PDZ domains: fundamental building blocks in the organization of protein complexes at the plasma membrane. J Clin Invest 103: 767-772.

Faulkner, G., A. Pallavicini, E. Formentin, A. Comelli, C. Ievolella, S. Trevisan, G. Bortoletto, P. Scannapieco, M. Salamon, V. Mouly, G. Valle, G. Lanfranchi (1999) ZASP: a new Zband alternatively spliced PDZ-motif protein. J Cell Biol 146: 465-475.

Franquemont, D.W., H.F. Frierson Jr, S.E. Mills (1991) An immunohistochemical study of normal endometrial stroma and endometrial stromal neoplasms. Evidence for smooth muscle differentiation. Am J Surg Pathol 15: 861870.

Getsios, S., G.T. Chen, M.D. Stephenson, P. Leclerc, O.W. Blaschuk, C.D. MacCalman (1998) Regulated expression of cadherin-6 and cadherin-11 in the glandular epithelial and stromal cells of the human endometrium. Dev Dyn 211: 238-247.

Glasser, S.R., J. Julian (1986) Intermediate filament protein as a marker of uterine stromal cell decidualization. Biol Reprod 35: 463-474.

Guy, P.M., D.A. Kenny, G.N. Gill (1999) The PDZ domain of the LIM protein enigma binds to $\beta$ tropomyosin. Mol Biol Cell 10: 1973-1984.

Harris, B.Z., A.W. Lim (2001) Mechanism and role of PDZ domains in signaling complex assembly. J Cell Sci 114: 3219-3231.
Jurata, L.W., G.N. Gill (1998) Structure and function of LIM domains. Curr Top Microbiol Immunol 228: 75-113.

Katoh, K., Y. Kano, M. Masuda, H. Onishi, K. Fujiwara (1998) Isolation and contraction of the stress fiber. Mol Biol Cell 9: 1919-1938.

Kiess, M., B. Scharm, A. Aguzzi, A. Hajnal, R. K1emenz, I. Schwarte-Waldhoff, R. Schafer (1995) Expression of ril, a novel LIM domain gene, is down-regulated in Hras-transformed cells and restored in phenotypic revertants. Oncogene 10: 61-68.

Kim, J.J., R.C. Jaffe, A.T. Fazleabas (1998) Comparative studies on the in vitro decidualization process in the baboon (Papio anubis) and human. Biol Reprod 59: 160-168.

Kimatrai, M., C. Oliver, A.C. Abadía-Molina, J.M. García-Pacheco, E.G. Olivares (2003) Contractile activity of human decidual stromal cells. J Clin Endocrinol Metab 88: 844-849.

Kotaka, M., S. Kostin, S.M. Ngai, K.K. Chan, Y.M. Lau, S.M. Lee, H. Li, E.K. Ng, J. Schaper, S.K. Tsui, K.P. Fung, C.Y. Lee, M.M. Waye (2000) Interaction of hCLIM1, an enigma family protein, with $\alpha$-actinin 2 . J Cell Biochem 78: 558-565.

Kuroda, S., C. Tokunaga, Y. Kiyohara, O. Higuchi, K. Hiroaki, K. Mizuno, G.N. Gill, U. Kikkawa (1996) Protein-protein interaction of zinc finger LIM domains with protein kinase C. J Biol Chem 271: 31029-31032.

MacCalman, C.D., E.E. Furth, A. Omigbodun, M. Bronner, C. Coutifaris, J.F. Strauss 3rd (1996) Regulated expression of cadherin-11 in human epithelial cells: a role for cadherin-11 in trophoblast-endometrium interactions? Dev Dyn 206: 201-211.

Mandai, K., H. Nakanishi, A. Satoh, H. Obaishi, M. Wada, H. Nishioka, M. Itoh, A. Mizoguchi, T. Aoki, T. Fujimoto, Y. Matsuda, S. Tsukita, Y. Takai (1997) Afadin: a novel actin filamentbinding protein with one PDZ domain localized at cadherin-based cell-to-cell adherens junction. J Cell Biol 139: 517-528.

Nakagawa, N., M. Hoshijima, M. Oyasu, N. Saito, K. Tanizawa, S. Kuroda (2000) ENH, containing PDZ and LIM domains, heart/skeletal muscle-specific protein, associates with cytoskeletal proteins through the PDZ domain. Biochem Biophys Res Commun 272: $505-$ 512.
Noyes, R.W., A.T. Hertig, J. Rock (1950) Dating the endometrial biopsy. Fertil Steril 1: 3-25.

Oliver, C., M.J. Montes, J.A. Galindo, C. Ruiz, E.G. Olivares (1999) Human decidual stromal cells express $\alpha$ smooth-muscle actin and show ultrastructural similarities with myofibroblasts. Hum Reprod 14: 1599-1605.

Rajasekaran AK, Rajasekaran SA (2003) Role of Na-K-ATPase in the assembly of tight junctions. Am J Physiol Renal Physiol 285: F388F396.

Salamonsen LA, Dimitriadis E, Jones RL, Nie G (2003) Complex regulation of decidualization: a role for cytokines and proteases - a review. Placenta 24(suppl A): S76-S85.

Vallenius, T., K. Luukko, T.P. Mäkelä (2000) CLP36 PDZ-LIM protein associates with nonmuscle $\alpha$-actinin- 1 and $\alpha$-actinin-4. J Biol Chem 275: $11100-11105$

Vallenius, T., B. Scharm, A. Vesikansa, K. Luukko, R. Schäfer, T. Mäkelä (2004) The PDZ-LIM protein RIL modulates actin stress fiber turnover and enhances the association of $\alpha$-actinin with actin. Exp Cell Res 293: 117-128.

Wang, H., D.C. Harrison-Shostak, J.J. Lemasters, B. Herman (1995) Cloning of a rat cDNA encoding a novel LIM domain protein with high homology to rat RIL. Gene 165: 267-271.

Wu, R.Y., G.N. Gill (1994) LIM domain recognition of a tyrosine-containing tight turn. J Biol Chem 269: 25085-25090.

Xia, H., S.T. Winokur, W.L. Kuo, M.R. Altherr, D.S. Bredt (1997) Actinin-associated LIM protein: identification of a domain interaction between PDZ and spectrin-like repeat motifs. J Cell Biol 139: 507-515.

Yoshimura, Y., K. Miyakoshi, T. Hamatani, K. Iwahashi, J. Takahashi, N. Kobayashi, K. Sueoka, T. Miyazaki, N. Kuji, M. Tanaka (1998) Role of $\beta_{1}$ integrins in human endometrium and decidua during implantation. Horm Res 50(suppl 2): 46-55.

Zhou, Q., P. Ruiz-Lozano, M.E. Martone, J. Chen (1999) Cypher, a striated muscle-restricted PDZ and LIM domain-containing protein, binds to alpha-actinin-2 and protein kinase $C$. J Biol Chem 274: 19807-19813. 